ENGINEERING CHANGE NOTICE

1. ECN 660497

Page 1 of $Z$

Proj.

ECN

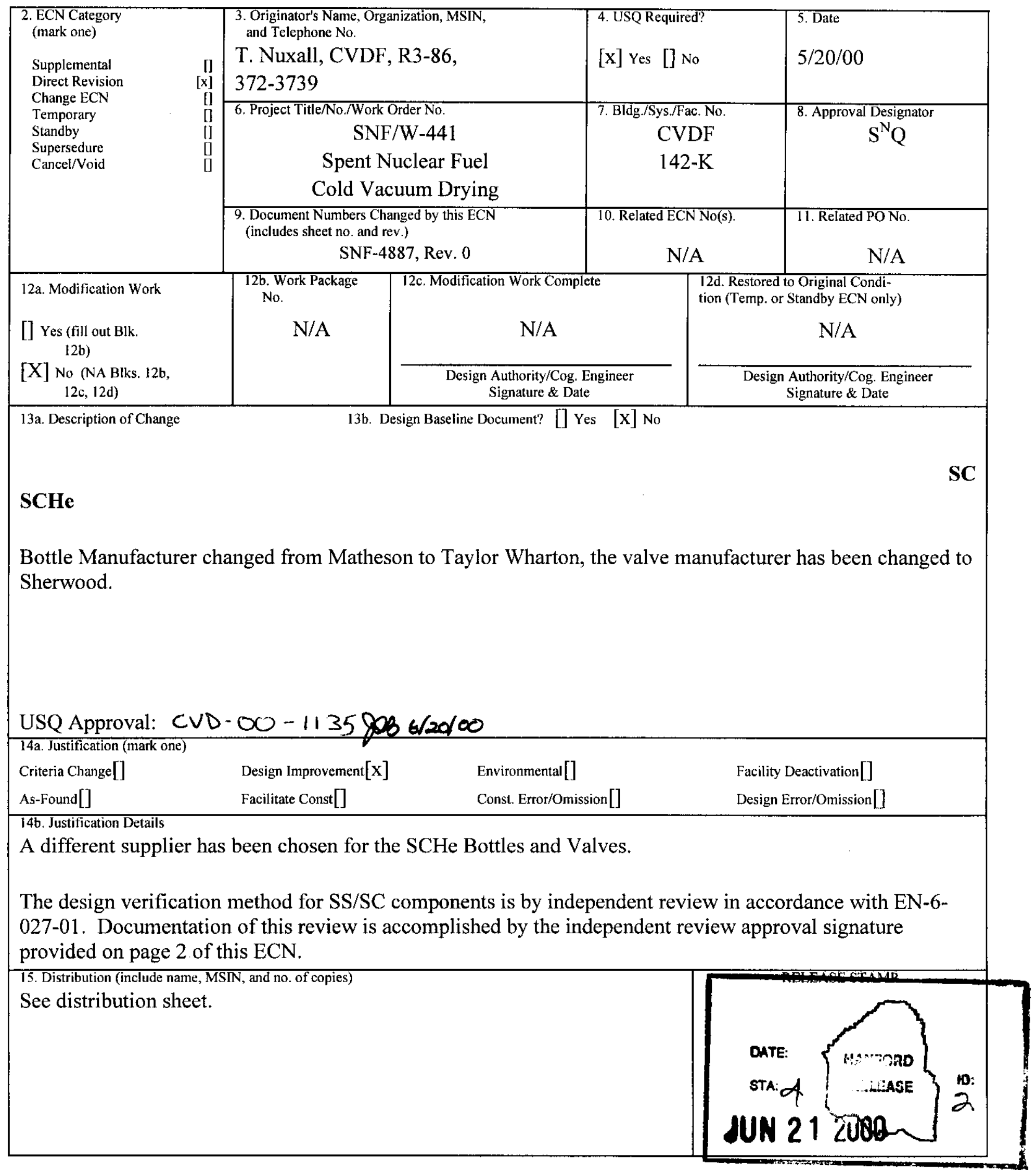




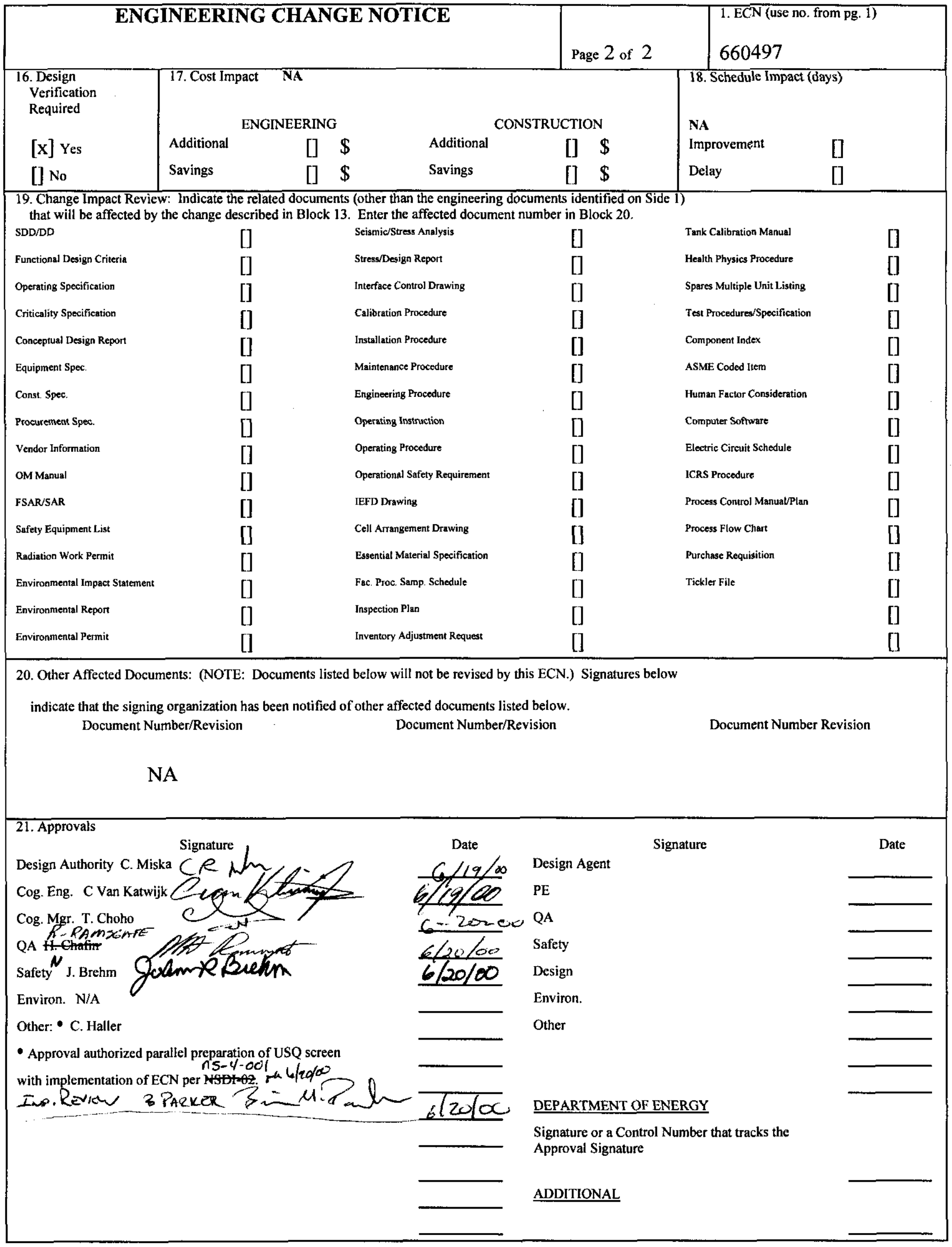


SNF-4887

Revision 1

\section{SCHe Helium Bottles and Associated Isolation Valves}

Prepared for the U.S. Department of Energy

Assistant Secretary for Environmental Management

Project Hanford Management Contractor for the

U.S. Department of Energy under Contract DE-AC06-96RL13200

Fluor Hanford

P.O. Box 1000

Richland, Washington 
SNF-4887

Revision 1

ECN 660497

\section{SCHe Helium Bottles and Associated Isolation Valves}

Project No: W-441

C Van Katwijk

$\mathrm{FH}$

Date Published

June 2000

Prepared for the U.S. Department of Energy

Assistant Secretary for Environmental Management

Project Hanford Management Contractor for the

U.S. Department of Energy under Contract DE-AC06-96RL13200

Fluor Hanford

P.O. Box 1000

Richland, Washington
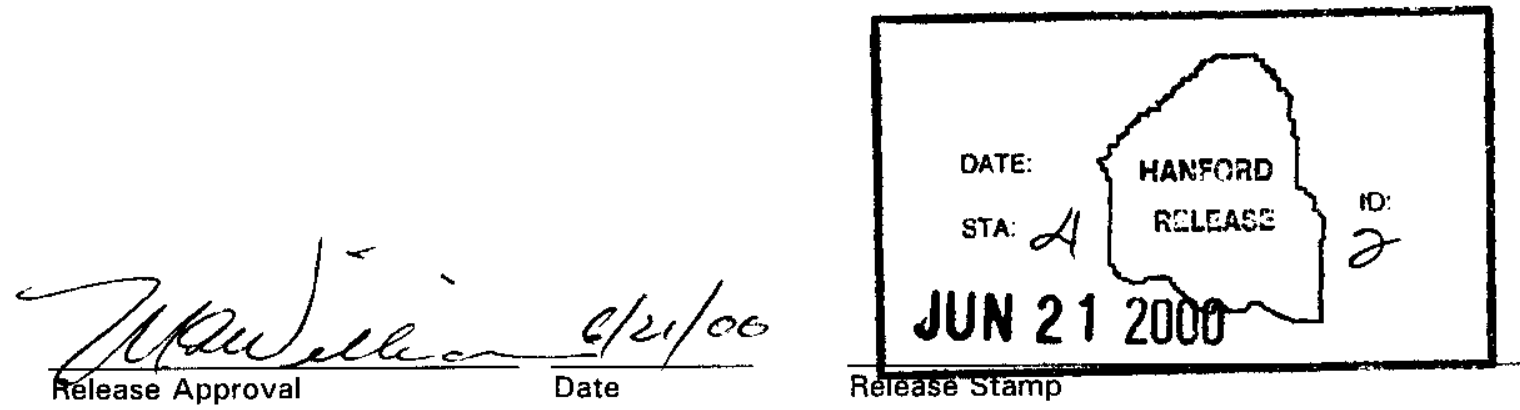
TRADEMARK DISCLAIMER

Reference herein to any specific commercial product, process,

or service by trade name, trademark, manufacturer, or

otherwise, does not necessarily constitute or imply its

endorsement, recommendation, or favoring by the United

States Government or any agency thereof or its contractors or subcontractors.

This report has been reproduced from the best available copy.

Printed in the United States of America

Total Pages: $\quad 13$

SNF-4887, new! 


\section{RECORD OF REVISION}

(2) Title

SCHe Helium Supply Bottles and Associated Isolation Valves

Change Control Record

$\begin{array}{ll}\text { (3) Revision } & \text { (4) Description of Change - Replace, Add, and Delete Pages }\end{array}$

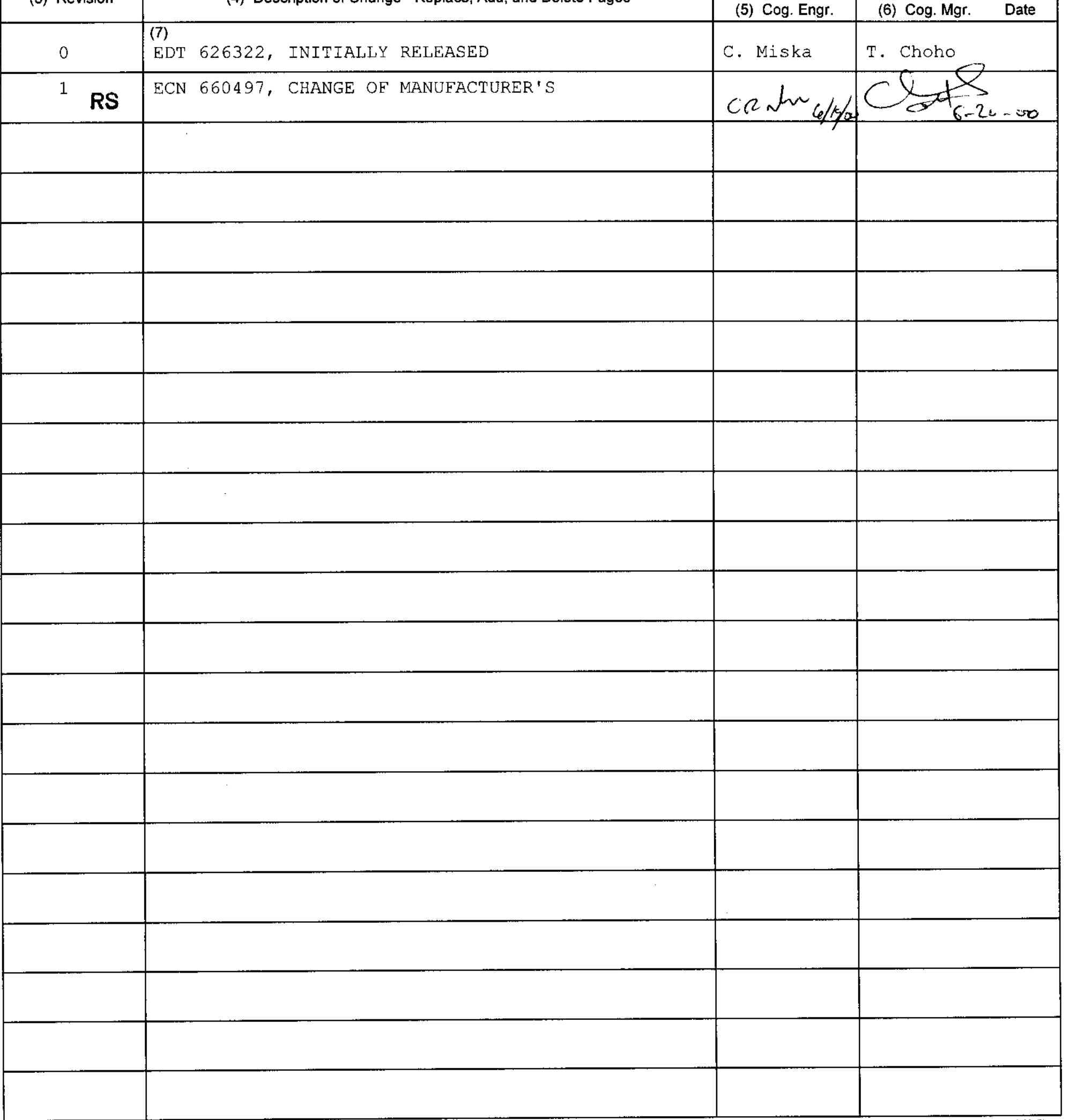




\begin{tabular}{|l|l|}
\cline { 2 - 2 } Commercial Grade Item Upgrade Dedication Form & SNF-4887, Rev. 1 \\
\hline ECN No. N/A CGI No. CGI-SNF-D-13-2-P5-050 & Page 1 of 10 \\
Title: SCHe Helium Supply Bottles and Associated Isolation Valves & \\
\hline
\end{tabular}

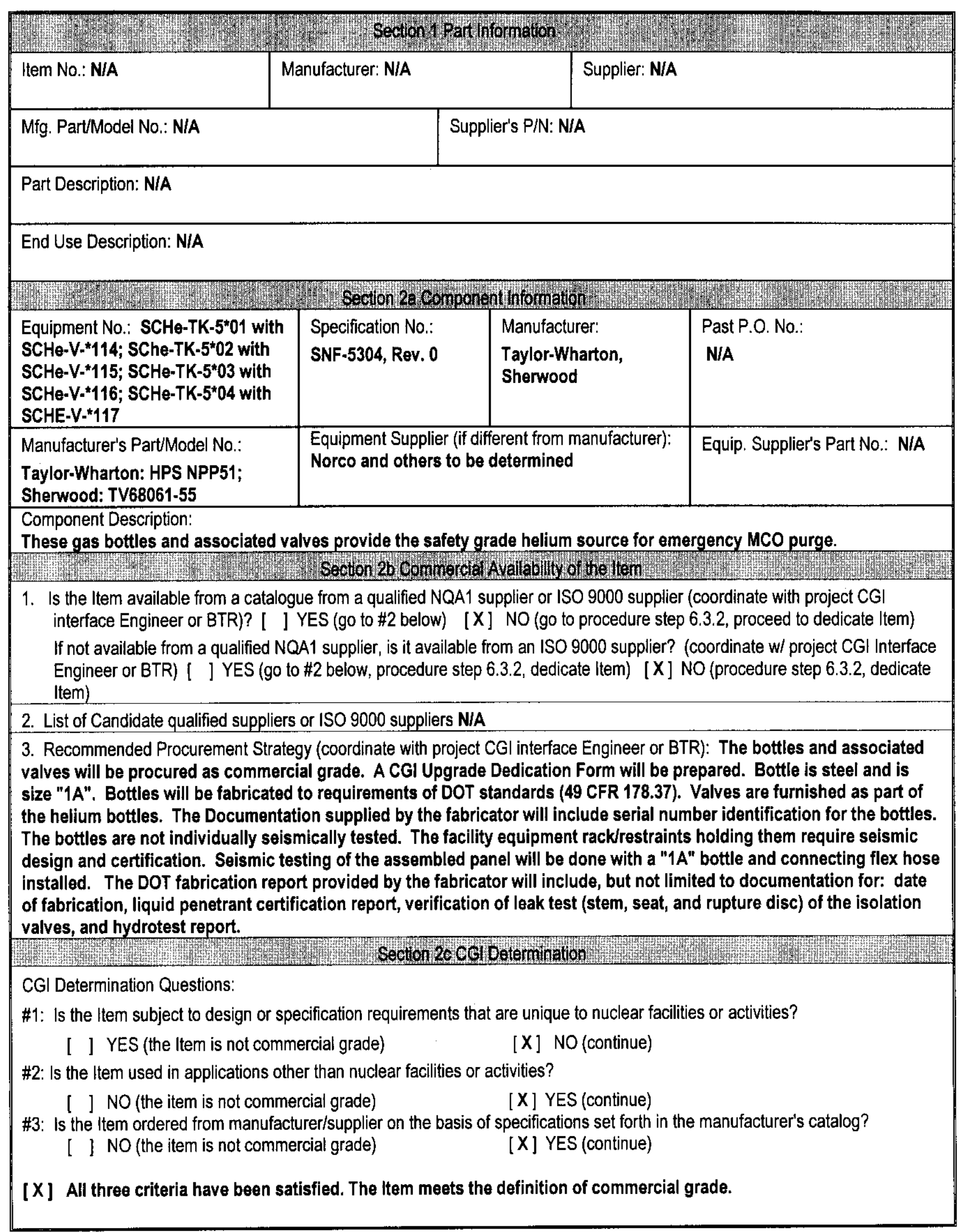


$X$ Item is being purchased from a non-ESL manufacturer supplier as CG to be used in a Safety Class application.

Item is being purchased from a non-ESL manufacturer supplier as CG to be used in a Safety Significant application.

Item was purchased from a non-ESL manufacturer supplier as CG to be used in a Safety Class application.

Item was purchased from a non-ESL manufacturer supplier as CG to be used in a Safety Significant application.

Other ('like-for-like', similar, substitution, replacement evaluation)

\section{Secion 3 Failure Eftects Evaluation}

A. Part/Component Safety Function:

1. SCHe Pressure Boundary Integrity- Prevents helium leakage from the $\mathrm{SCHe}$ System.

2. Maintain critical function before and after Seismic event.

3. Supply Helium for MCO emergency helium purge.

B. Part/Component Functional Mode:

Safety Function \#1: [ ] Active [X] Passive

Safety Function \#2: [ ] Active [X] Passive

Safety Function \#3: [ ] Active [X] Passive

Active - Mechanical or Electrical change of state is required to occur for the component to perform its safety function

Passive - Change of state is not required for the component to perform its safety function

C. Host Component Safety Function (if applicable): N/A

D. Failure Mode(s) and the effects on component or system safety function (see Worksheet 1):

1. Fracture of the pressure boundary or of the piping connection resulting in loss of helium from the SCHe supply.

2. Foreign matter introduced during bottle shipment and change out could block flow in the hose or in the regulator. H.

Environmental Qualification Required:

Yes [ ]

No [ $\mathbf{X}$ ] Environmental Condition B

Natural Phenomena Hazard (NPH) Design Required:

Yes [X] HNF-PRO-97, Rev. 0; SNF-5304, Rev. 0

No []
If yes: Environmental Qualification Requirements

Limiting Environmental Conditions:

Required Safety Functions:

Qualification Period:

If yes: NPH Design Requirements

Performance Category: PC-3

NPH Design Req'ts.: Seismic Condition A

Required Safety Functions: Pressure Boundary Integrity; Maintain critical function before and after Seismic Event.

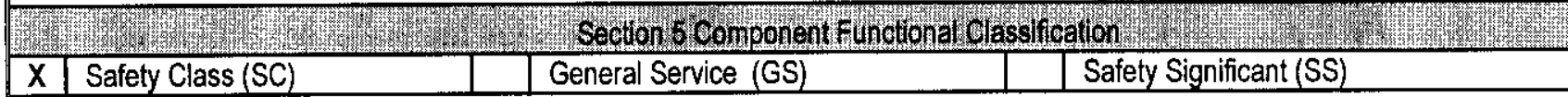

If part/component classification is different from host component/system, document basis. N/A

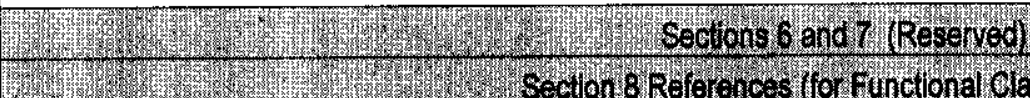

W Sectons Reforences (or functional cassificajion)

National Codes/Standards: DOT 49 CFR 178.37

Safety Analysis Report (SAR): HNF-3553, Rev. OA, Annex B

Drawings: H-1-82165, Rev.2; HNF-SD-SNF-SEL-002, Rev.7

Vendor Manual/Manufacturer/Supplier Information: Norco Technical Information. 
Commercial Grade Item Upgrade Dedication Form

ECN No. N/A CGI No. CGI-SNF-D-13-2-P5-050

Title: SCHe Helium Supply Bottles and Associated Isolation Valves

SNF-4887, Rev. 1

Page 3 of 10

\begin{tabular}{|c|c|c|c|c|}
\hline Cmicar Cnaraclerisics & 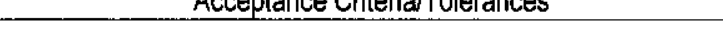 & Acc Metn & (1) & Function. \\
\hline \multicolumn{5}{|l|}{ Bottle } \\
\hline \multicolumn{5}{|c|}{ 1. Item Identification Critical Characteristics (necessary for reasonable assurance that the Item delivered is the Item specified) } \\
\hline $\begin{array}{l}\text { Construction } \\
\text { Documentation for: DOT } 49 \\
\text { CFR } 178.37 \\
\text { DOT-3AA3600 \& } \\
\text { TC-3AAM154BAR }\end{array}$ & $\begin{array}{l}\text { Fabrication Report information including: serial } \\
\text { numbers, date of fabrication, liquid penetrant } \\
\text { certification report, verification of leak test (stem, } \\
\text { seat, and rupture disc) of the isolation valves, and } \\
\text { hydrotest report. All items acceptable. }\end{array}$ & $1, \mathrm{IN}$ & $\mathrm{X}$ & \\
\hline Nameplate - Manufacturer & Taylor-Wharton & $1, \mathbf{I N}$ & $\mathbf{X}$ & \\
\hline Bottle Size & Nominal 9" Diameter X 51" Height & 1, IN & $\mathbf{X}$ & \\
\hline $\begin{array}{l}\text { Procurement and/or Model } \\
\text { No. }\end{array}$ & Bottle: Taylor-Wharton & $1, \mathrm{IN}$ & $\bar{X}$ & \\
\hline \multicolumn{5}{|c|}{ 2. Physical Critical Characteristics (for reasonable assurance that the Item delivered is the Item specified) } \\
\hline Material, Bottle & Steel (Note 3) & $1, \mathbb{I N} ; 1, T$ & $\underline{x}$ & \\
\hline \multicolumn{5}{|c|}{ Valve } \\
\hline \multicolumn{5}{|c|}{ 1. Item Identification Critical Characteristics (necessary for reasonable assurance that the Item delivered is the Item specified) } \\
\hline Nameplate - Manufacturer & Sherwood & \begin{tabular}{l|l}
, $\mathrm{IN}$ \\
\end{tabular} & $\mathrm{X}$ & \\
\hline $\begin{array}{l}\text { Procurement and/or Model } \\
\text { No. }\end{array}$ & TV 68061-55 & $1, \mathbb{I N}$ & $\bar{X}$ & \\
\hline \multicolumn{5}{|c|}{ 2. Physical Critical Characteristics (for reasonable assurance that the Item delivered is the Item specified) } \\
\hline Material, Valve Body & Brass & $1, \mathrm{IN}$ & $\mathbf{X}$ & \\
\hline Size & Inlet: 3/4"-14 NGT - Tapered; Outlet: CGA 680 & $1, \mathrm{IN}$ & $\bar{X}$ & \\
\hline \multicolumn{5}{|c|}{3 Performance Critical Characteristics (for reasonable assurance that the Item will perform its intended safety function(s)) } \\
\hline Valve Seat Leakage & $\begin{array}{l}\text { ASME Section V, Article10 - Sensitive Leak Test - } \\
\text { Pressure Test to } 3000 \text { psig (No Leakage-No } \\
\text { Bubbles) }\end{array}$ & $1, T$ & & $\mathrm{x}$ \\
\hline \multicolumn{5}{|l|}{ Bottle \& Valve: } \\
\hline \multicolumn{5}{|c|}{3 Performance Critical Characteristics (for reasonable assurance that the Item will perform its intended safety function(s)) } \\
\hline Environmental & Note 1 & & & \\
\hline Seismic Condition A Event & Note 2 & $1, T$ & & $\mathrm{X}$ \\
\hline \multicolumn{3}{|c|}{$\begin{array}{l}\text { 4. Notes and Legend: } \\
\text { Rev. 1: Revised bottle manufacturer to Taylor-Wharton, Isolation Valve to Sherwood. } \\
\text { Rev. 2: Revised Pressure test to be done at } 3000 \text {. } \\
\text { 1. The helium bottle and valve are not subject to degradation at ambient conditions of } 40^{\circ} \mathrm{F} \text { and } \\
60 \% \text { RH or } 115^{\circ} \mathrm{F} \text { and } 22 \% \mathrm{RH} \text { and is suitable for Environmental Condition B application. } \\
\text { 2. Maintain critical function before and after Seismic event. W-441-P5, Rev. 2, Appendix I, page } \\
\text { I-2, provides a seismic testing plan for these components at a seismic spectra TBD. } \\
\text { Equipment that has been shaker-table tested should not be installed in a plant (Ref. IEEE } \\
\text { Standard } 344-1987 \text {, Section 7). Consequently, the seismic test constitutes a destructive test. } \\
\text { 3. Material verification acceptance method may be by either inspection or test. }\end{array}$} & \multicolumn{2}{|c|}{$\begin{array}{l}\text { Acceptance Method (Acc } \\
\text { Meth): } \\
\text { 1.Special Test and Inspection: } \\
\text { Y,IN for Inspection } \\
\text { 1.T Ior Test } \\
\text { 2. Commercial Grade Survey } \\
\text { 3. Source Verification } \\
\text { 4. Vendorittem History }\end{array}$} \\
\hline 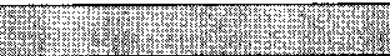 & - Hection 10 in tial Review and Approval & & & \\
\hline
\end{tabular}


Commercial Grade Item Upgrade Dedication Form

ECN No. N/A _ CGI No. CGI-SNF-D-13-2.P5-050

SNF-4887, Rev. 1

Title: $\mathrm{SCHe}$ Helium Supply Bottles and Associated Isolation Valves

Page 4 of 10

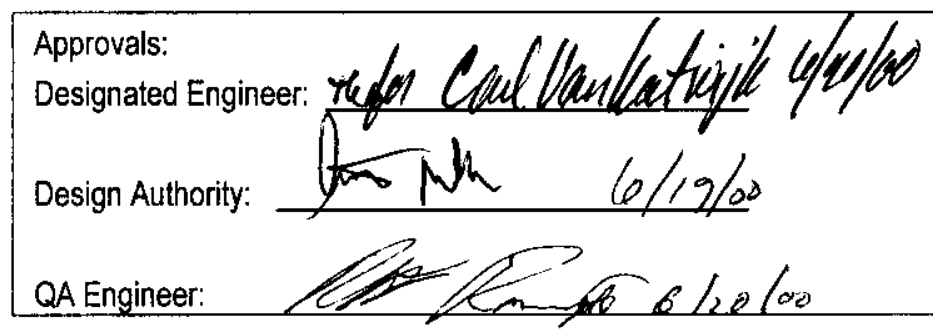




\begin{tabular}{l|l|}
\cline { 2 - 2 } Commercial Grade Item Upgrade Dedication Form & SNF-4887, Rev. 1 \\
\hline ECN No. N/A CGI No. CGI-SNF-D-13-2-P5-050 & Page 5 of 10 \\
Title: SCHe Helium Supply Bottles and Associated Isolation Valves & \\
\hline
\end{tabular}

\begin{tabular}{|c|c|c|c|}
\hline \multicolumn{4}{|c|}{$\begin{array}{c}\text { WORKSHEET } 1 \\
\text { DETERMINATION OF FAILURE MECHANISMS }\end{array}$} \\
\hline \multicolumn{4}{|c|}{ WW } \\
\hline Typical Failure & \multirow[t]{2}{*}{ Definition } & \multicolumn{2}{|c|}{$\mathbf{X}=$ Applicable to Component under Evaluation } \\
\hline & & $\mathbf{X} ?$ & $\begin{array}{l}\text { Indicate Failure Mode } \\
\end{array}$ \\
\hline Fracture & $\begin{array}{l}\text { Separation of a solid accompanied by little or no } \\
\text { macroscopic plastic deformation. }\end{array}$ & $x$ & $\begin{array}{l}\text { Fracture of the bottle or the connection would fail the } \\
\text { pressure boundary and stop the supply of emergency } \\
\text { helium to that purge path. }\end{array}$ \\
\hline Corrosion & $\begin{array}{l}\text { The gradual deterioration of a material due to chemical } \\
\text { or electrochemical reactions, such as oxidation, between } \\
\text { the material and its environment. }\end{array}$ & & \\
\hline Erosion & $\begin{array}{l}\text { Destruction of materials by the abrasive action of } \\
\text { moving fluids, usually accelerated by the presence of } \\
\text { solid particles carried with the fluid. }\end{array}$ & & \\
\hline Open Circuit & $\begin{array}{l}\text { An electrical circuit that is unintentionally broken so that } \\
\text { there is no complete path for current flow. }\end{array}$ & & \\
\hline Short Circuit & $\begin{array}{l}\text { An abnomal connection by which an electrical current is } \\
\text { connected to ground, or to some conducting body, } \\
\text { resulting in excessive current flow. }\end{array}$ & & \\
\hline Blockage & $\begin{array}{l}\text { Clogging of a filtering medium resulting in the inability to } \\
\text { perform its purification function or blockage of flow. }\end{array}$ & $X$ & $\begin{array}{l}\text { Foreign matter introduced during bottle changeout could } \\
\text { block flow in the valve. }\end{array}$ \\
\hline Seizure & $\begin{array}{l}\text { Binding of a normally moving item through excessive } \\
\text { pressure, temperature, friction, jamming. }\end{array}$ & & \\
\hline $\begin{array}{l}\text { Unacceptable } \\
\text { Vibration }\end{array}$ & $\begin{array}{l}\text { Mechanical oscillations produced are beyond the } \\
\text { defined permissible limits due to unbalancing, poor } \\
\text { support, or rotation at critical speeds. }\end{array}$ & & \\
\hline Loss of Properties & $\begin{array}{l}\text { A loss of mechanical and physical properties of a } \\
\text { material due to exposure to high temperatures, radiation } \\
\text { exposure. }\end{array}$ & & \\
\hline Excess Strain & $\begin{array}{l}\text { Under the action of excessive external forces the } \\
\text { material of the part has been deformed or distorted. }\end{array}$ & & \\
\hline Mechanical Creep & $\begin{array}{l}\text { From prolonged exposure to high temperature and } \\
\text { stress, the object will show a slow change in its physical } \\
\text { (shape and dimension) and mechanical characteristics. }\end{array}$ & & \\
\hline Ductile Fracture & $\begin{array}{l}\text { Fracture characterized by tearing of metal accompanied } \\
\text { by appreciable gross plastic deformation. }\end{array}$ & & \\
\hline & Secton 2 dedinenal Folutre Modes app & of & tho component Under mvatuaton \\
\hline oreian & introduced inside the hose during bottle & nor & at could block flow in the hose or in the regulator. \\
\hline
\end{tabular}


Commercial Grade Item Upgrade Dedication Form

ECN No. N/A CGI No. CGI-SNF-D-13-2-P5-050

Title: SCHe Helium Supply Bottles and Associated Isolation Valves

SNF-4887, Rev. 1

Checklist 1 - Acceptance Method 1 - Special Test/Inspection Verification

\begin{tabular}{|c|c|c|c|c|c|}
\hline \multirow{2}{*}{\multicolumn{4}{|c|}{$\begin{array}{l}\text { Item Description: } \\
\text { SCHe Helium Supply Bottles and Associated Valves } \\
\text { System \#: 13-2 } \\
\text { Model \#: Taylor-Wharton: HPS NPP51 }\end{array}$}} & \multirow{2}{*}{\multicolumn{2}{|c|}{$\begin{array}{l}\text { SECTION I } \\
\text { Equip \#: SCHe-TK-5*01 with SCHe-V-*114 } \\
\text { SChe-TK-5*02 with SCHe-V-*115 } \\
\text { SCHe-TK-5*03 with SCHe-V-*116 } \\
\text { SCHe-TK-5*04 with SCHE-V-*117 }\end{array}$}} \\
\hline & & & & & \\
\hline \multicolumn{3}{|c|}{$\begin{array}{l}\text { Manufacturer } \\
\text { (Address/Phone): } \\
\text { Taylor - Wharton } \\
521 \text { Green Cove Rd } \\
\text { Huntsville, AL 35803 } \\
\text { (256)650-9100; P.O.: } \\
11638-3\end{array}$} & $\begin{array}{l}\text { Sherwood } \\
2111 \text { Liberty Drive, } \\
\text { Niagara Falls, NY } 14304\end{array}$ & $\begin{array}{l}\text { Supplier } \\
\text { (Address/Phone): }\end{array}$ & $\begin{array}{l}\text { NorLab } \\
1121 \text { W. Amity, } \\
\text { Boise, ID } 83705 \\
(208) 336-1643\end{array}$ \\
\hline \multicolumn{6}{|c|}{ SECTION 2 CRITLAL CHARACTERISTICS TO EE VERIFIED BY NETHOO 1} \\
\hline \multicolumn{6}{|c|}{\begin{tabular}{|cccc} 
Insp & Test & $\begin{array}{c}\text { Post } \\
\text { Test }\end{array}$ \\
\end{tabular}} \\
\hline $\mathrm{x}$ & & \multicolumn{4}{|c|}{ Construction Documentation } \\
\hline $\mathbf{x}$ & & \multicolumn{4}{|c|}{ Nameplate - Manufacturer } \\
\hline$x$ & & \multicolumn{4}{|c|}{ Bottle Size } \\
\hline$x$ & & \multicolumn{4}{|c|}{ Procurement and/or Model No. (Bottle and Valve) } \\
\hline $\mathrm{x}$ & $\bar{x}$ & \multicolumn{4}{|c|}{ Material, Bottle (Verification may be by either inspection or test) } \\
\hline $\mathbf{x}$ & $\bar{x}$ & \multicolumn{4}{|c|}{ Material, Valve (Verification may be by either inspection or test) } \\
\hline $\mathrm{x}$ & & \multicolumn{4}{|c|}{ Size, Valve } \\
\hline $\mathrm{x}$ & & \multicolumn{4}{|c|}{ Pressure Boundary Integrity (See Documentation) } \\
\hline & $\mathbf{x}$ & \multicolumn{4}{|c|}{ Seismic Condition A } \\
\hline & $\bar{x}$ & \multicolumn{4}{|c|}{ Valve Leakage } \\
\hline & \multicolumn{5}{|c|}{ 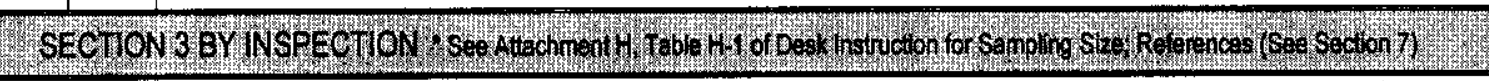 } \\
\hline \multicolumn{6}{|c|}{ Bottle } \\
\hline \multicolumn{6}{|c|}{$\begin{array}{l}\text { Characteristic: Construction Documentation } \\
\text { Acceptance Criteria: Fabrication report including: serial numbers, date of fabrication, liquid penetrant certification, } \\
\text { verification of leak test (stem, seat, and rupture disc) of the isolation valves, and hydrotest certification. All items } \\
\text { acceptable. } \\
\text { Receipt Inspection Plan / Report \#: }\end{array}$} \\
\hline \multicolumn{5}{|c|}{$\begin{array}{l}\text { Characteristic: Nameplate - Manufacturer } \\
\text { Acceptance Criteria: Taylor-Wharton } \\
\text { Receipt Inspection Plan / Report\#: }\end{array}$} & Sample Size ${ }^{*}: 100 \%$ \\
\hline \multicolumn{6}{|c|}{$\begin{array}{l}\text { Characteristic: Bottle Size } \\
\text { Acceptance Criteria: Nominal 9" Diameter X 51" Height } \\
\text { Receipt Inspection Plan / Report \#: }\end{array}$} \\
\hline \multicolumn{6}{|c|}{$\begin{array}{l}\text { Characteristic: Procurement and/or Model No. } \\
\text { Acceptance Criteria: Bottle: Taylor-Wharton } \\
\text { Receipt Inspection Plan / Report \#: }\end{array}$} \\
\hline
\end{tabular}




\begin{tabular}{|l|l|}
\cline { 2 - 2 } Commercial Grade Item Upgrade Dedication Form & SNF-4887, Rev. I \\
\hline ECN No. N/A CGI No. CGI-SNF-D-13-2-P5-050 & Page 7 of 10 \\
Title: SCHe Helium Supply Bottles and Associated Isolation Valves & \\
\hline
\end{tabular}

Characteristic: Material, Bottle

Sample Size*: $100 \%$

Acceptance Criteria: Steel

Receipt Inspection Plan / Report \#:

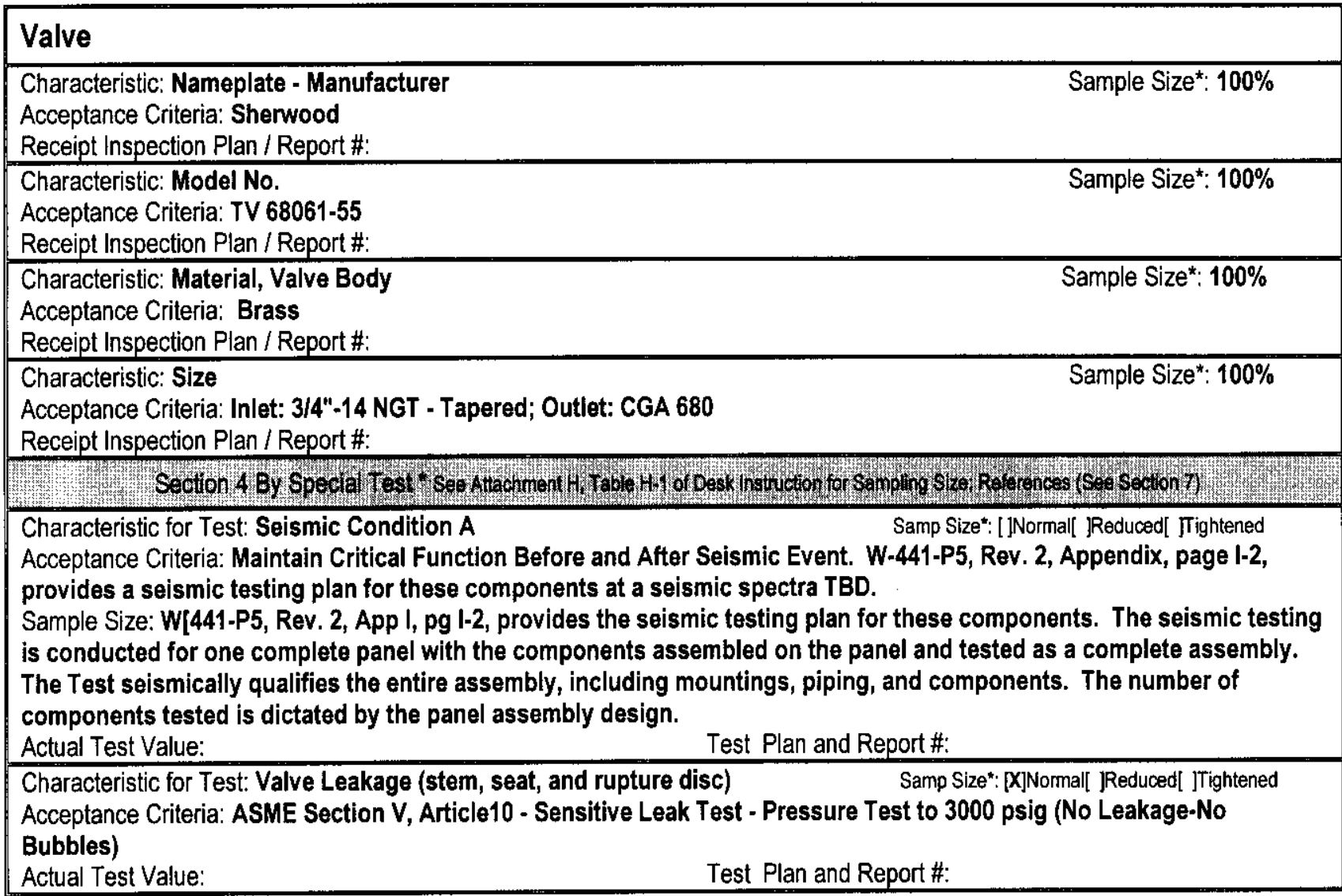




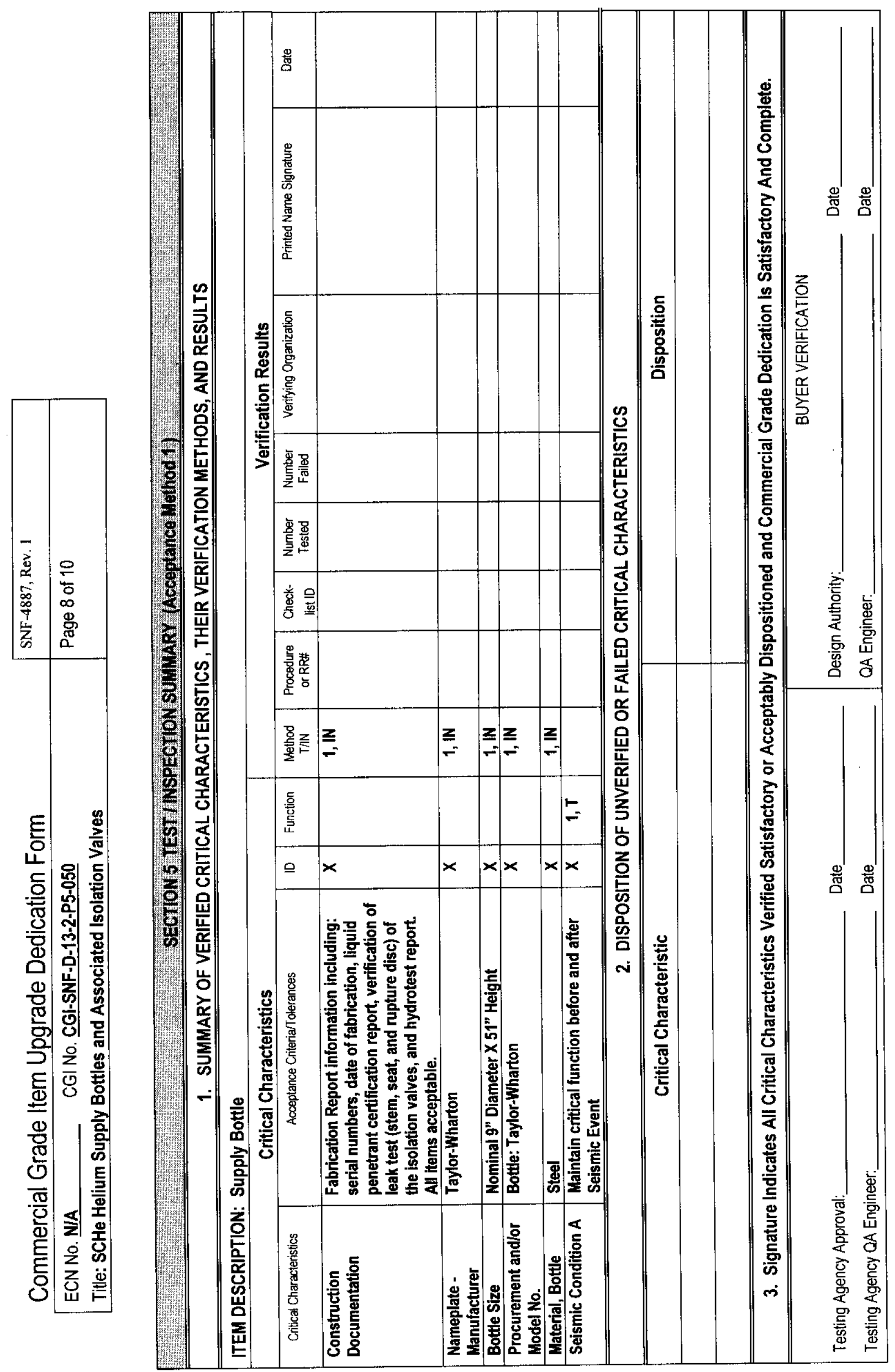




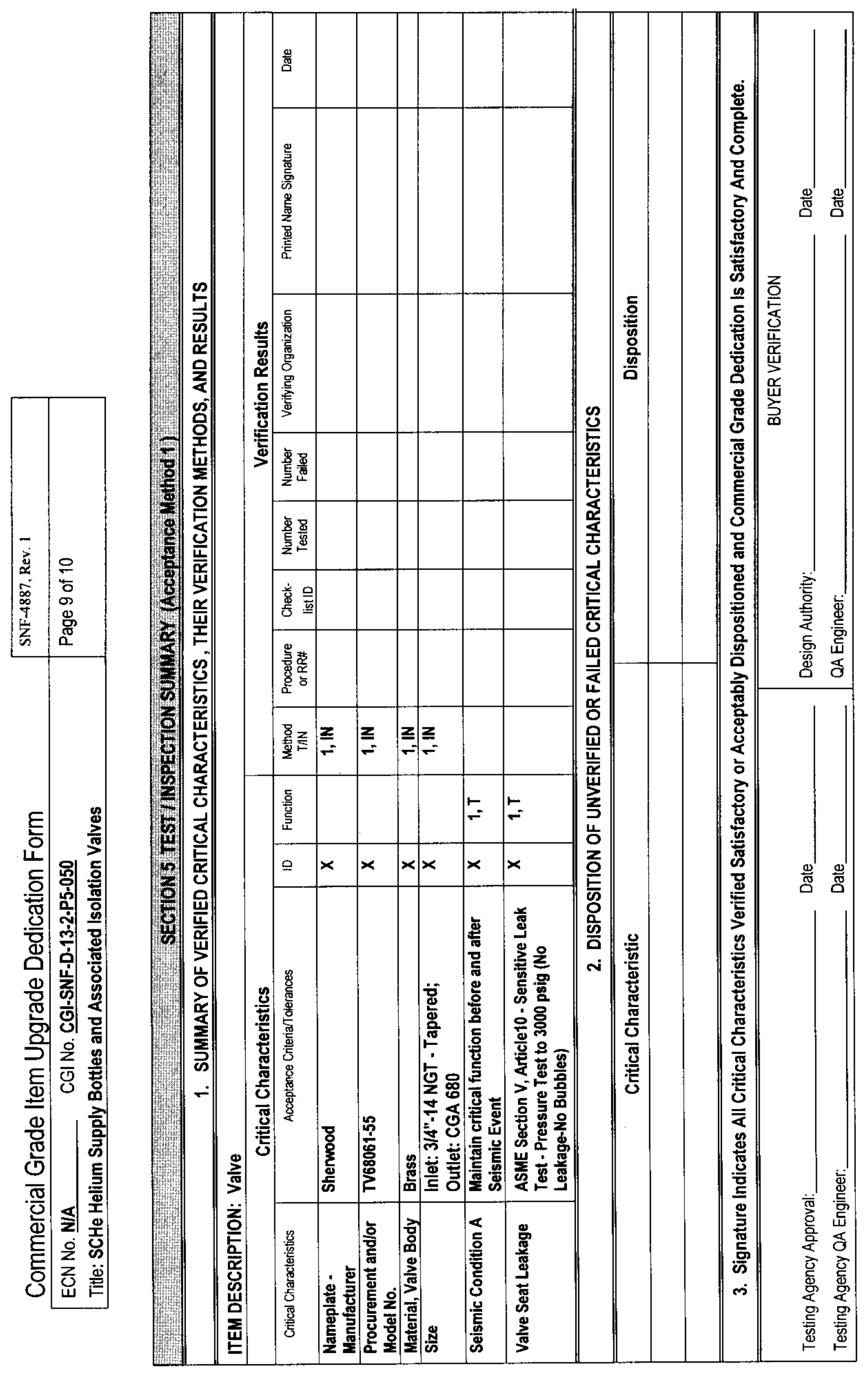


Commercial Grade Item Upgrade Dedication Form

ECN No. N/A CGI No. CGI-SNF-D-13-2-P5-050

Title: SCHe Helium Supply Bottles and Associated Isolation Valves

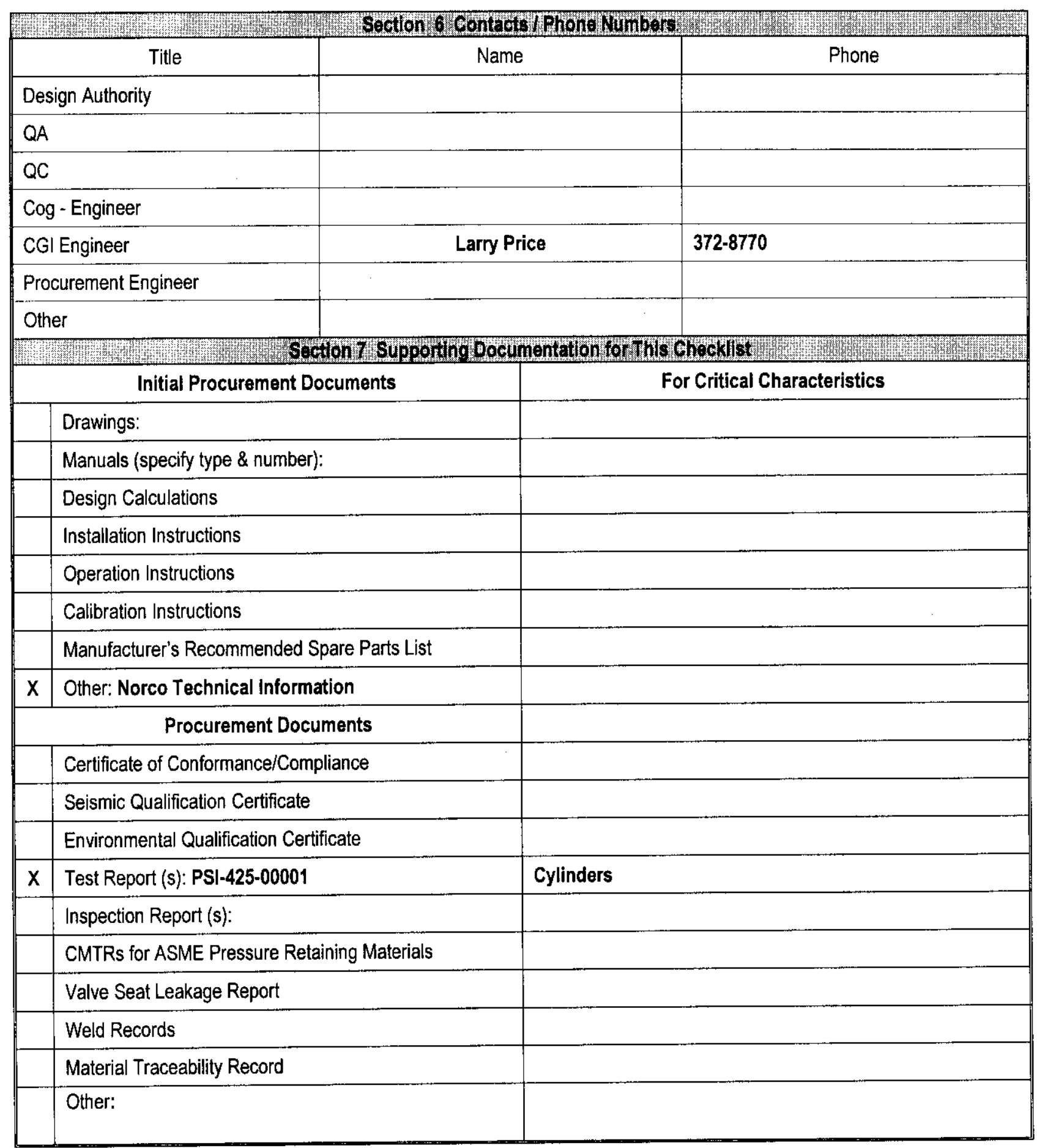




\section{DISTRIBUTION SHEET}

To

Distribution

Project Title/Work Order

W-441, CGI Package P5-050

\section{Name}

D. Whitehurst

G. Singh

A. Artzer (CVD Library)

R. Ramsgate

J. Brehm

P. Beaudet

P. Morrell (AVS)

M. Evarts (AI)

L. Price

SNE Startup

SNF Project Files

SNF Satelite Library

C. Van Katwijk

D. Whitworth

T. Nuxall

C. Miska
From

SNE-CVD
Page 1 of 1

Date 6/19/00

EDT No. NA

ECN No. 660497

\begin{tabular}{|c|c|c|c|c|} 
MSIN & $\begin{array}{c}\text { Text } \\
\text { With All } \\
\text { Attach. }\end{array}$ & Text Only & $\begin{array}{c}\text { Attach./ } \\
\text { Appendix } \\
\text { Only }\end{array}$ & $\begin{array}{c}\text { EDT/ECN } \\
\text { Only }\end{array}$ \\
\hline
\end{tabular}

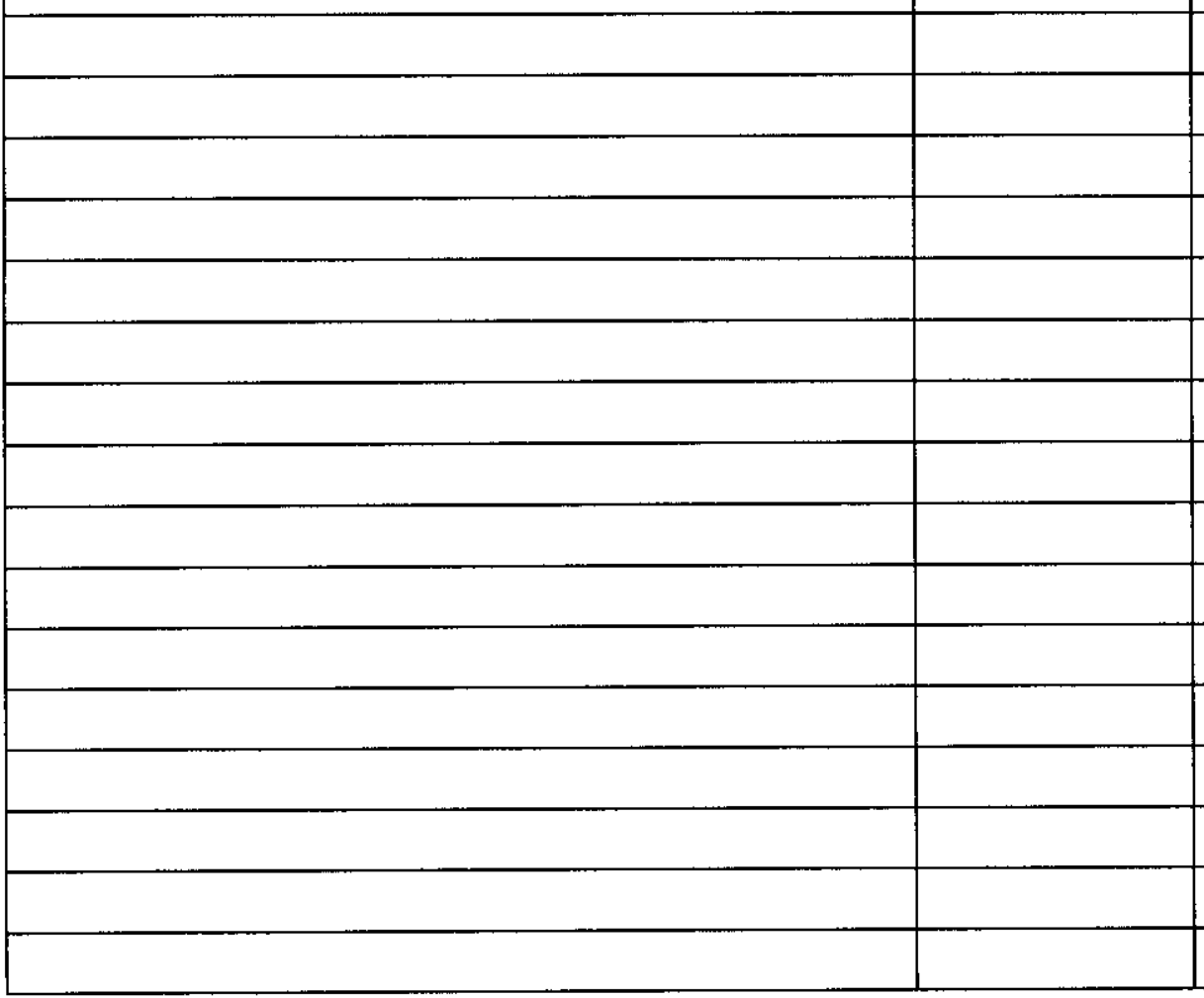

$\times 3-74$

$\times 3-74$

R3-86

$\times 3-74$

R3-26

58-07

G1-50

N1-29

R3-26

B2-64

R3-11

X3-25

R3-47

R3-11

X3-74

$\times 3-74$

$\mathrm{x}$

$\dot{x}$

$\mathrm{x}$

$\mathrm{x}$

$\mathrm{x}$

$x$

$\mathrm{x}$

$x$

$x$

$\mathrm{x}$

$\mathrm{x}$

$\mathrm{x}$

\begin{tabular}{|l}
\hline$x$ \\
\hline$x$ \\
\hline$x$ \\
\hline$x$ \\
\hline$x$ \\
\hline$x$ \\
\hline$x$ \\
\hline$x$ \\
\hline$x$ \\
\hline$x$ \\
\hline$x$ \\
\hline$x$ \\
\hline$x$ \\
\hline$x$ \\
\hline$x$ \\
\hline
\end{tabular}

\begin{tabular}{|l}
\hline$x$ \\
\hline$x$ \\
\hline$x$ \\
\hline$x$ \\
\hline$x$ \\
\hline$x$ \\
\hline$x$ \\
\hline$x$ \\
\hline$x$ \\
\hline$x$ \\
\hline$x$ \\
\hline$x$ \\
\hline$x$ \\
\hline$x$ \\
\hline$x$ \\
\hline
\end{tabular}

\begin{tabular}{|l}
\hline$x$ \\
\hline$x$ \\
\hline$x$ \\
\hline$x$ \\
\hline$x$ \\
\hline$x$ \\
\hline$x$ \\
\hline$x$ \\
\hline$x$ \\
\hline$x$ \\
\hline$x$ \\
\hline$x$ \\
\hline$x$ \\
\hline$x$ \\
\hline$x$ \\
\hline
\end{tabular}

\begin{tabular}{|l}
\hline$x$ \\
\hline$x$ \\
\hline$x$ \\
\hline$x$ \\
\hline$x$ \\
\hline$x$ \\
\hline$x$ \\
\hline$x$ \\
\hline$x$ \\
\hline$x$ \\
\hline$x$ \\
\hline$x$ \\
\hline$x$ \\
\hline$x$ \\
\hline$x$ \\
\hline
\end{tabular}

$x$ 